\title{
Effects of Superabsorbent Polymers on the Hydraulic Parameters and Water Retention Properties of Soil
}

\author{
Renkuan Liao, ${ }^{1}$ Wenyong Wu, ${ }^{1}$ Shumei Ren, ${ }^{2}$ and Peiling Yang ${ }^{2}$ \\ ${ }^{1}$ State Key Laboratory of Simulation and Regulation of Water Cycles in River Basins, \\ China Institute of Water Resources and Hydropower Research, Beijing 100048, China \\ ${ }^{2}$ College of Water Conservancy and Civil Engineering, China Agricultural University, Beijing 100083, China
}

Correspondence should be addressed to Peiling Yang; yangpeiling@126.com

Received 30 January 2016; Revised 16 May 2016; Accepted 5 June 2016

Academic Editor: João Garcia

Copyright (C) 2016 Renkuan Liao et al. This is an open access article distributed under the Creative Commons Attribution License, which permits unrestricted use, distribution, and reproduction in any medium, provided the original work is properly cited.

\begin{abstract}
Superabsorbent polymers (SAPs) are widely applied in dryland agriculture. However, their functional property of repeated absorption and release of soil water exerts periodic effects on the hydraulic parameters and water-retention properties of soil, and as this property gradually diminishes with time, its effects tend to be unstable. During the 120-day continuous soil cultivation experiment described in this paper, horizontal soil column infiltration and high-speed centrifugation tests were conducted on SAP-treated soil to measure unsaturated diffusivity $D$ and soil water characteristic curves. The experimental results suggest that the SAP increased the water retaining capacity of soil sections where the suction pressure was between 0 and $3,000 \mathrm{~cm}$. The SAP significantly obstructed water diffusion in the soil in the early days of the experiment, but the effect gradually decreased in the later period. The average decrease in water diffusivity in the treatment groups fell from $76.6 \%$ at 0 days to $1.2 \%$ at 120 days. This research also provided parameters of time-varying functions that describe the unsaturated diffusivity $D$ and unsaturated hydraulic conductivity $K$ of soils under the effects of SAPs; in future research, these functions can be used to construct water movement models applicable to SAP-treated soil.
\end{abstract}

\section{Introduction}

The application of superabsorbent polymers (SAPs) for the purpose of enhancing soil water retention represents an important nonengineering water conservation technique for dryland farming. This technique is widely used in producing crops such as apples, grapes, wheat, and maize [1-4], and it has proven to be effective in saving water and increasing yields. In many farming areas with limited water supplies, crop growth relies completely on rainwater. However, the uneven spatiotemporal distribution of precipitation and the soil's poor ability to conserve moisture keep rainwater use efficiency low in these areas, exerting a direct impact on crop growth [5-7]. Applying SAPs to the soil is effective in improving rainwater use efficiency in dryland farming areas $[8,9]$, because SAPs can repeatedly absorb and retain rainwater entering the soil to reduce deep seepage losses and then gradually release the water to the plants as the soil dries and the plants' root pressure increases. This mechanism ensures a continuous water supply for plants during their growth periods [10, 11].

After treatment with SAPs, soil demonstrates remarkable changes in its hydraulic parameters and water holding properties. Han et al. [12] found that the periodic absorption and release of water by SAPs exert time-varying effects on the soil's properties, causing the hydraulic parameters in SAP-treated soil to vary irregularly with time. Bai et al. [13] discovered that, during wet-dry soil cycles, the application of SAPs can reduce soil's bulk density, with a higher SAP dosage producing a greater effect. Other studies [14-17] have revealed that the repeated water absorption and release mechanism of SAPs not only ensures water supply for plants, but also alters the pattern of soil water movement by influencing the soil's mechanical and chemical properties, local microbial communities, and root growth. This adds to the difficulty and complexity inherent in modeling water movement in soil to which SAPs have been applied. 


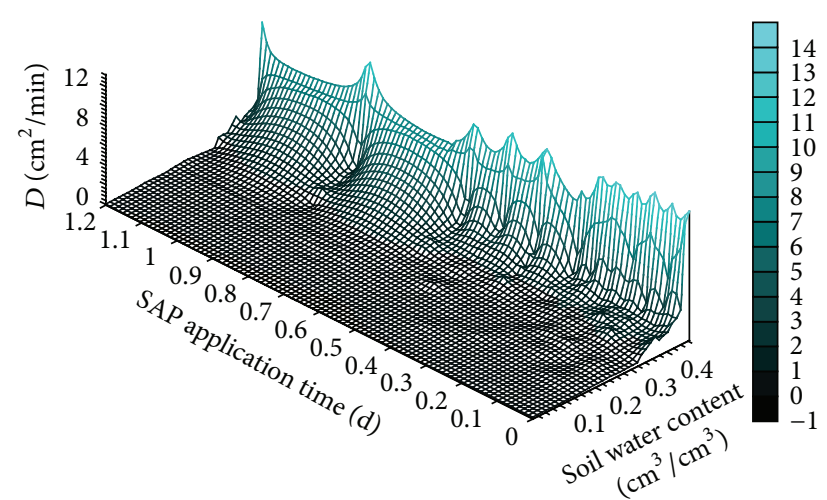

(a)

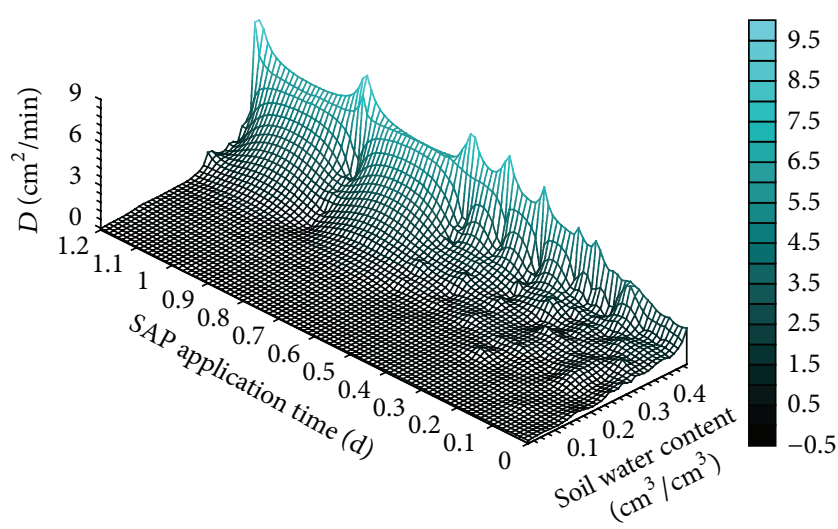

(c)

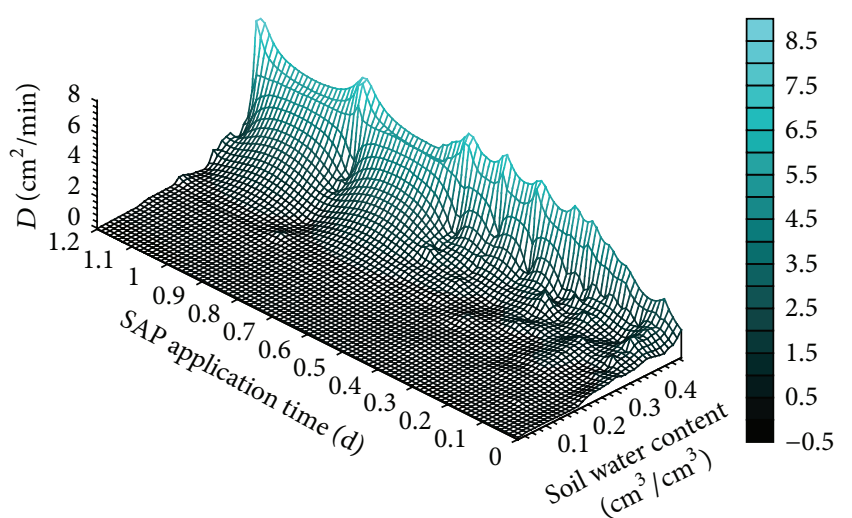

(b)

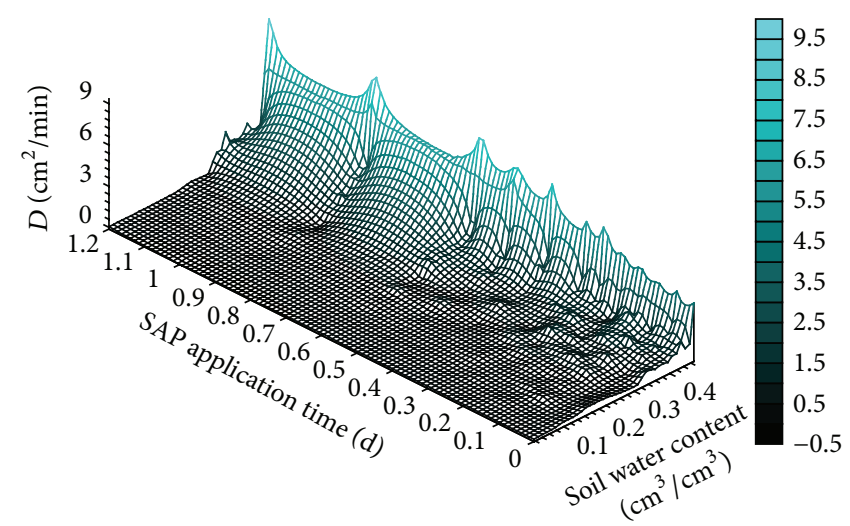

(d)

FIGURE 1: 3D unsaturated diffusivity at different times. (a) Control group; (b) P-S1; (c) P-S2; (d) P-S3. Note: the SAP application time at a point is equal to its $y$-axis value multiplied by 100 .

Accurate characterization of soil's hydraulic parameters and water retention properties is a key step in modeling water movement [18-20]. However, only a few studies have provided quantitative descriptions of the dynamic characteristics of SAP-treated soil's hydraulic parameters [12, 21, 22]. The dynamic effects of SAPs on soil's hydraulic parameters and water retention properties are not yet clear, obstructing indepth research into water movement models that are applicable to SAP-treated soils, as well as other related research.

In this context, a 120-day soil cultivation experiment was carried out, with horizontal soil column infiltration and high-speed centrifugation tests conducted to investigate the patterns of dynamic variation in the hydraulic parameters and water holding properties of SAP-treated soil. Timevarying functions were obtained to describe the unsaturated diffusivity and unsaturated hydraulic conductivity of soil, providing a basis for constructing relevant models, such as a model of soil water movement under the influence of SAPs.

\section{Methods}

2.1. Materials. This experiment tested a cross-linked soil SAP with particle sizes between 0.02 and $0.05 \mathrm{~mm}$, manufactured from polyacrylamide and acrylic acid by Dongying Huaye New Material Co., Ltd., in Shandong. The soil tested was sand loam containing $52.4 \%$ sand, $36.1 \%$ silt, and $11.5 \%$ clay, taken from topsoil in the greenhouses and fields at the International Seed Industry Park in Yujiawu Town, Tongzhou District, Beijing. In terms of nutrients, the tested soil contained total nitrogen of $1.06 \pm 0.11 \mathrm{~g} / \mathrm{kg}$, available nitrogen of $47.20 \pm$ $0.14 \mathrm{mg} / \mathrm{kg}$, total phosphorus of $0.74 \pm 0.01 \mathrm{~g} / \mathrm{kg}$, available phosphorus of $26.45 \pm 2.33 \mathrm{mg} / \mathrm{kg}$, total potassium of $18.65 \pm$ $1.34 \mathrm{~g} / \mathrm{kg}$, rapidly available potassium of $106.00 \pm 5.66 \mathrm{mg} / \mathrm{kg}$, and organic content of $10.85 \pm 0.21 \mathrm{~g} / \mathrm{kg}$. The soil was cultivated in round polyvinyl chloride pots with a height of $35 \mathrm{~cm}$ and a diameter of $25 \mathrm{~cm}$.

\subsection{Process}

2.2.1. Treatment. The soil was divided into a control group and three treatment groups. No SAP was applied to the soil in the control sample. In the first treatment group, labeled $\mathrm{P}-\mathrm{S} 1, \mathrm{SAP}$ at a polymer concentration of $0.06 \%$ was applied to the soil. In the second treatment group, P-S2, 0.03\% SAP was applied to the soil. In the third treatment group, P-S3, the soil was treated with $0.01 \%$ SAP. Each treatment process was repeated 12 times.

2.2.2. Methods. First, the SAP was evenly mixed with airdried soil. The mixtures were then put into pots and 


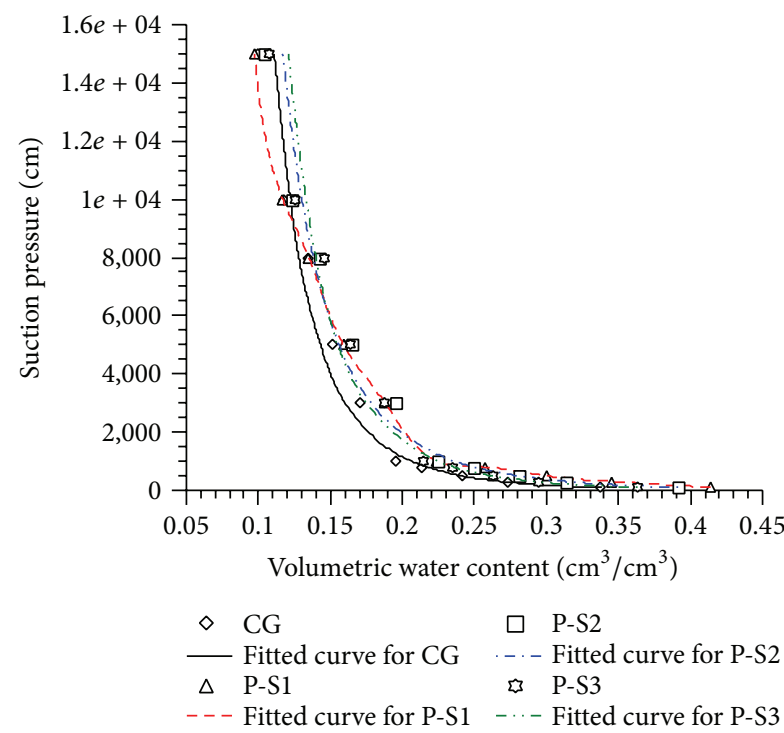

(a)

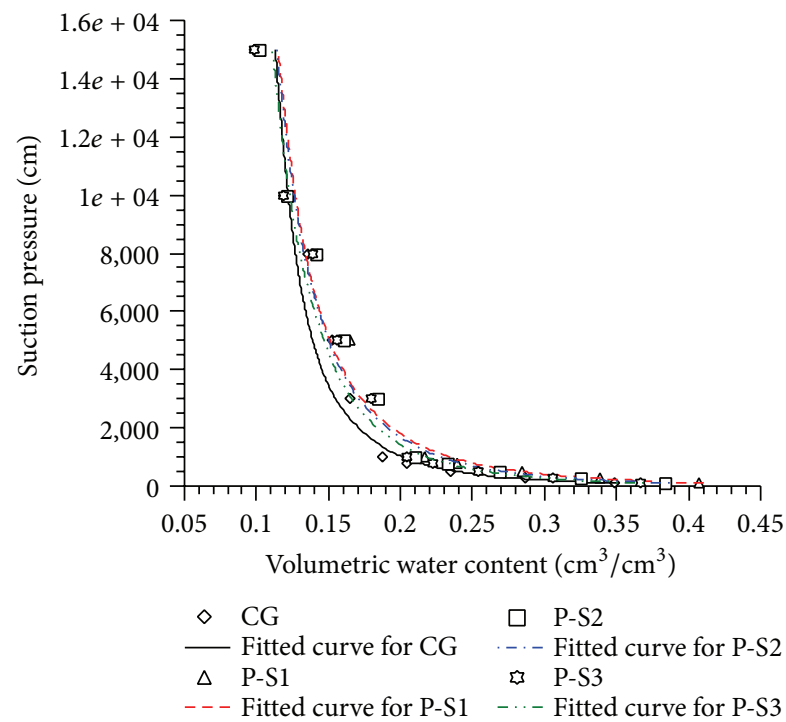

(c)

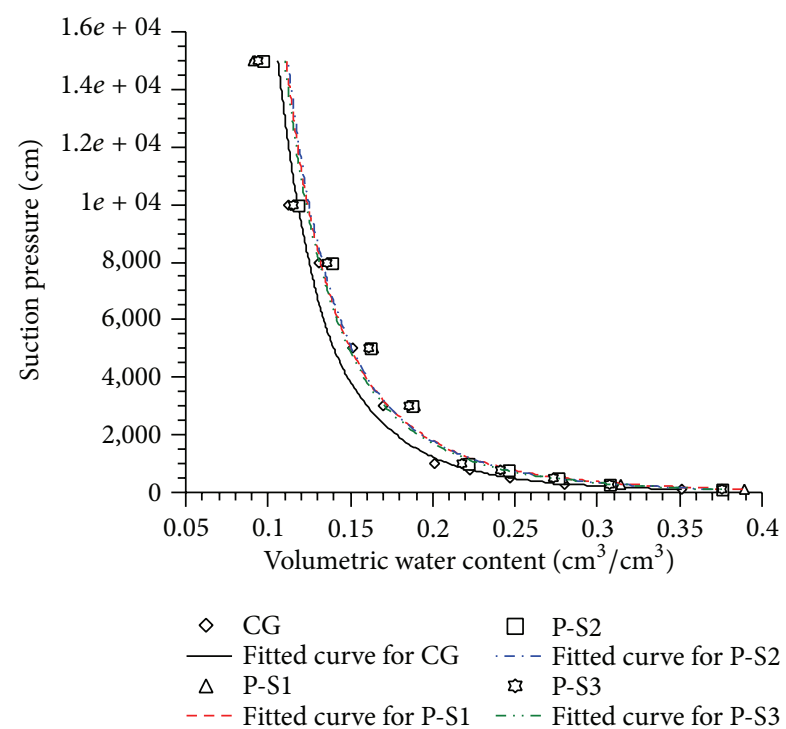

(e)

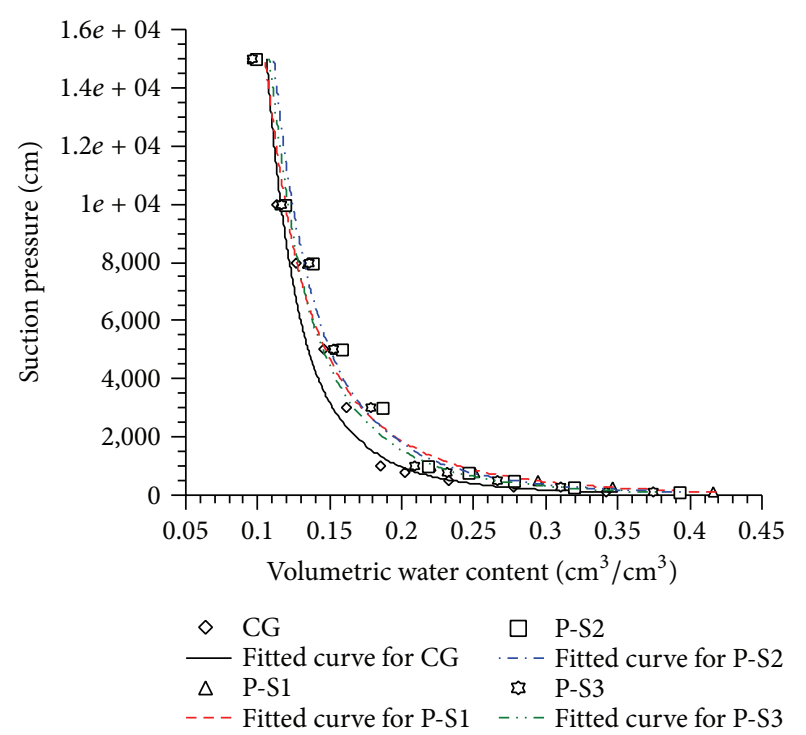

(b)

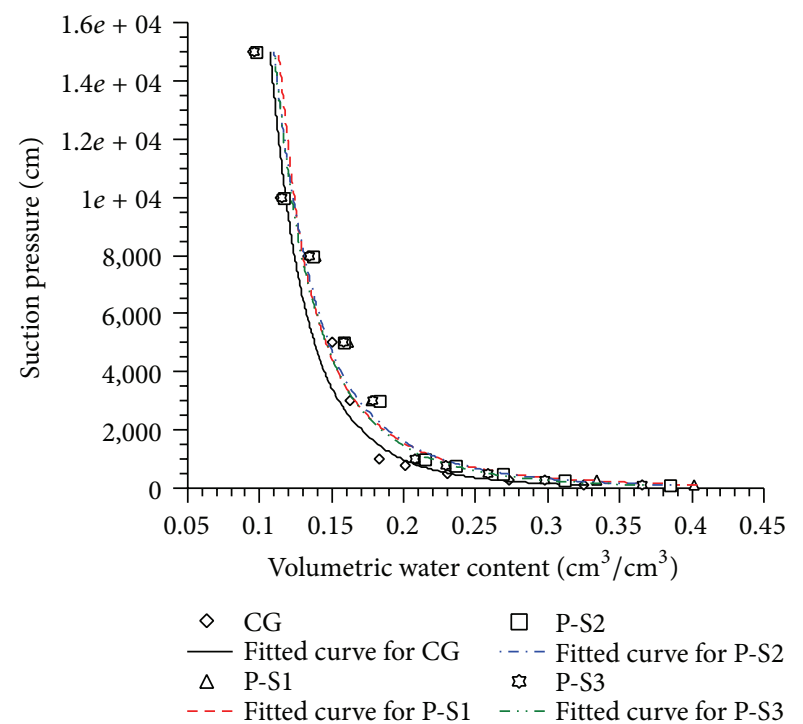

(d)

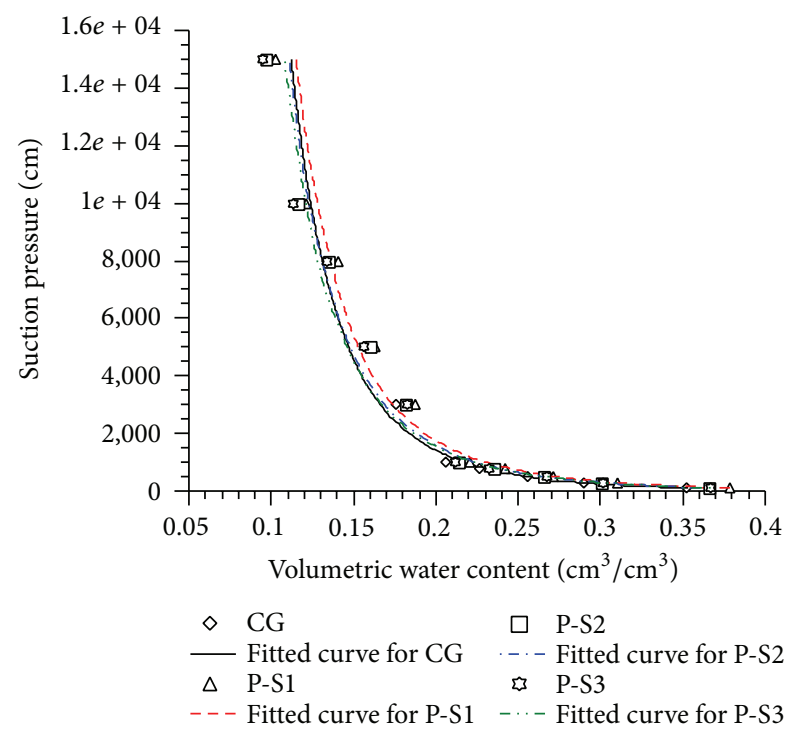

(f)

Figure 2: Soil water characteristic curves at different times. (a) $T=0 \mathrm{~d}$; (b) $T=15 \mathrm{~d}$; (c) $T=30 \mathrm{~d}$; (d) $T=50 \mathrm{~d}$; (e) $T=90 \mathrm{~d}$; (f) $T=120 \mathrm{~d}$. 


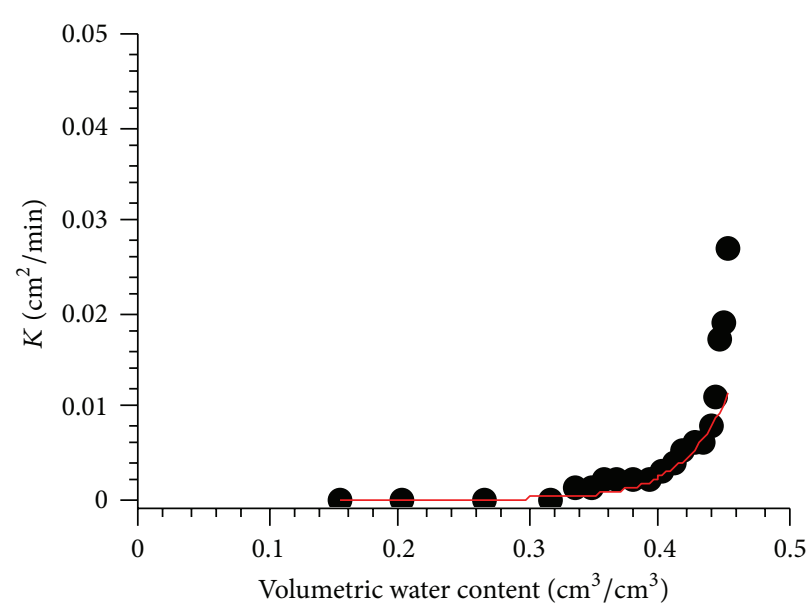

(a)

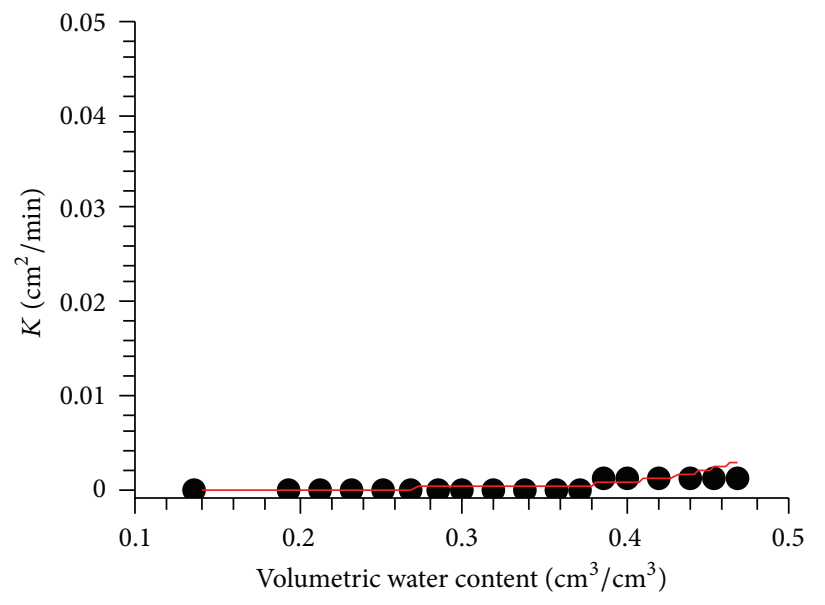

(c)

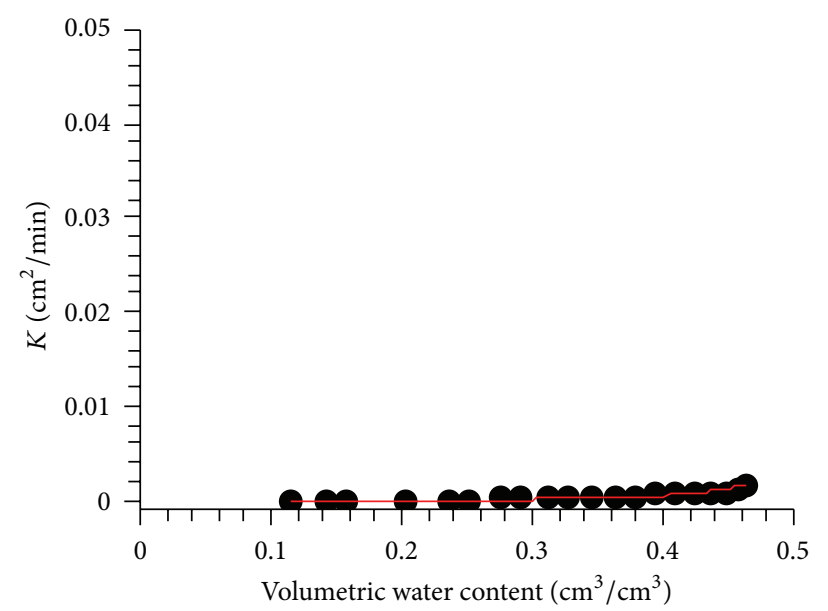

(b)

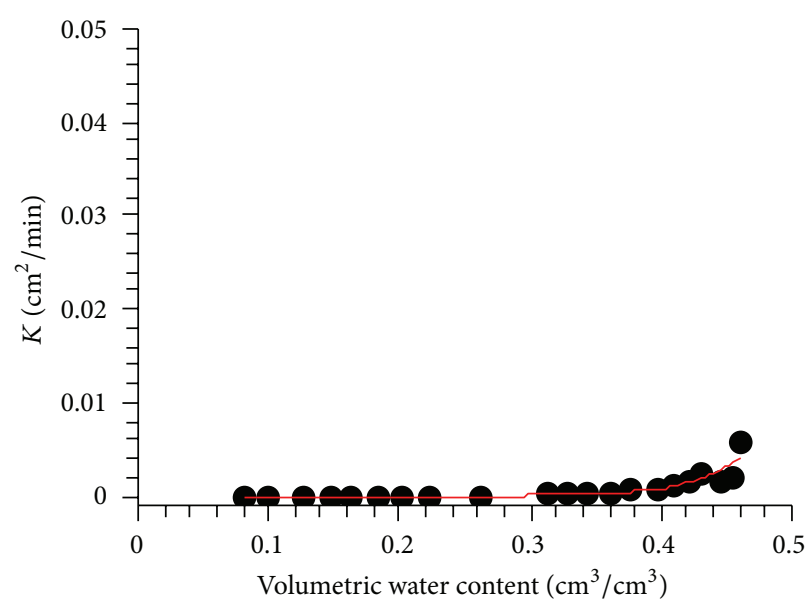

(d)

FIgUre 3: Unsaturated hydraulic conductivity of the four groups at 0 days. (a) Control group; (b) P-S1; (c) P-S2; (d) P-S3.

compacted layer by layer, with each layer being 4 to $5 \mathrm{~cm}$ thick, to achieve a bulk density of $1.28 \mathrm{~g} / \mathrm{cm}^{3}$. At the bottom of each pot, under the soil mixtures, a $2 \mathrm{~cm}$ thick filter bed of gravel was laid upon the drainage hole. After the soil thickness in the pots reached $28 \mathrm{~cm}$, a $2 \mathrm{~cm}$ thick layer of quartz sand was applied on the soil surface to reduce evaporation, leaving a $3 \mathrm{~cm}$ hydraulic head above the quartz sand for irrigation. After these preparations, the field capacity (FC) of the soil was measured using the cutting ring method, yielding a result of $0.37 \mathrm{~cm}^{3} / \mathrm{cm}^{3}$. Every two to three days, four pots were randomly chosen from each sample group and weighed to measure average soil water content. During the experiment, the soil's volumetric water content was maintained between $60 \%$ and $100 \%$ of FC. If the volumetric water content of the soil in a pot neared $60 \%$ of FC, the soil was irrigated until the value reached $100 \%$ of FC.

2.3. Sampling and Testing. Soil in the pots was sampled using cutting rings at $0,5,10,15,20,25,30,40,50,60,90$, and 120 days. Part of each soil sample was packaged in filter paper and tested in the lab using a high-speed centrifugation method to analyze the water characteristic curves. After the remainders of the sampled soils were air-dried, a horizontal soil column infiltration test was conducted to measure their unsaturated diffusivity [12]. Soil's unsaturated hydraulic conductivity can be calculated from the unsaturated diffusivity measurement according to the following relationships: $\theta=\theta_{r}+\left(\theta_{s}-\theta_{r}\right) /[1+$ $\left.(\alpha h)^{n}\right]^{m}$ and $K(\theta)=C(\theta) D(\theta)$.

2.4. Data Analysis. The software RETC analyzed the soil water characteristic data to infer the values of unsaturated hydraulic conductivity. The parameters of time-varying functions for unsaturated diffusivity and unsaturated hydraulic conductivity were obtained by $\mathrm{R}$ programming and curve fitting.

\section{Results and Analysis}

3.1. Effect of SAP on Unsaturated Soil Water Diffusivity. Figure 1 displays the three-dimensional distribution of unsaturated diffusivity for each of the four groups at $0,5,10,15$, $20,25,30,40,50,60,90$, and 120 days. The unsaturated diffusivity of the control group changed slightly throughout the experiment, and the population variance of the control 


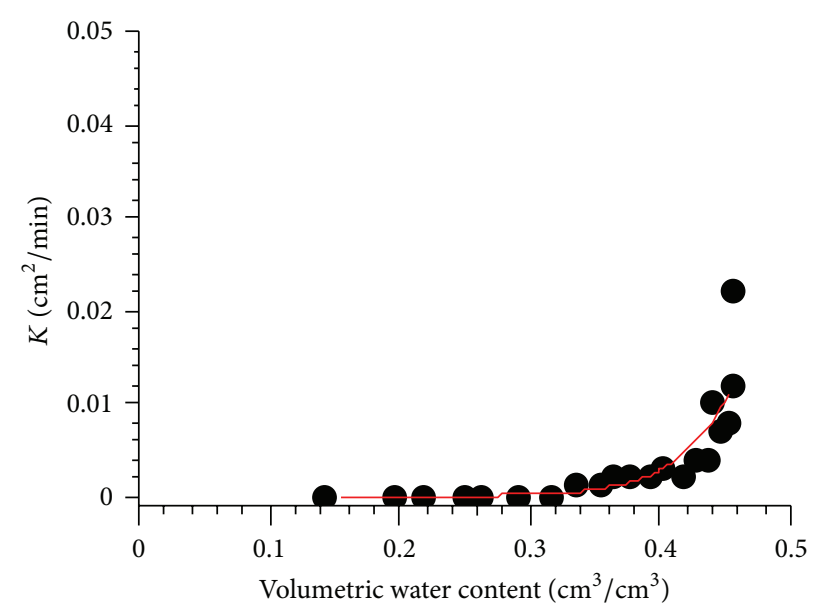

(a)

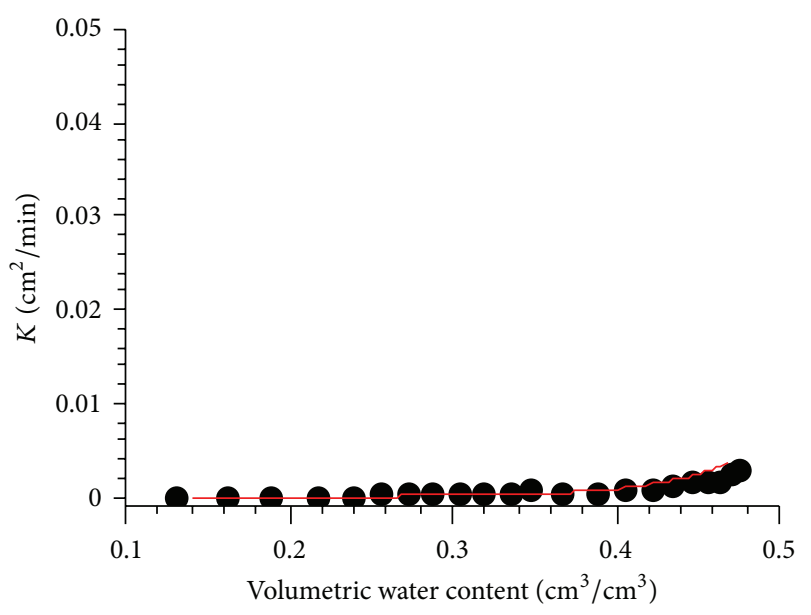

(c)

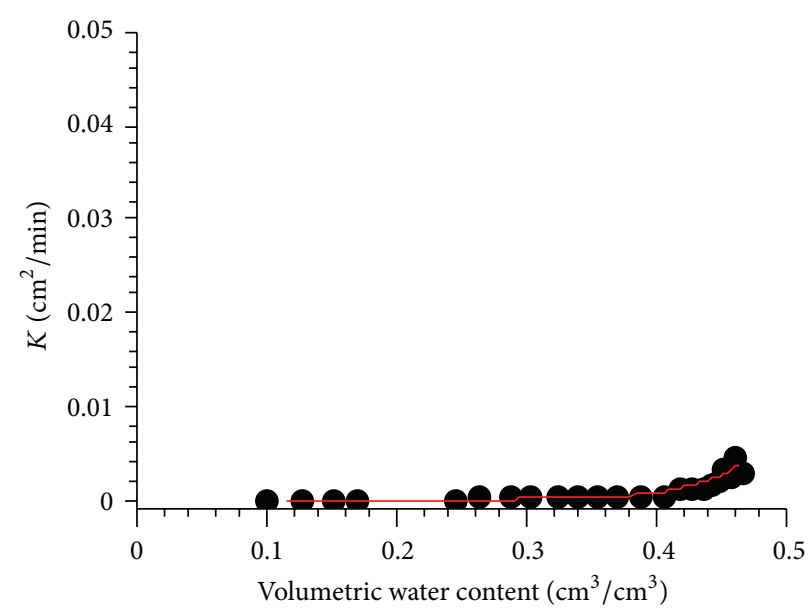

(b)

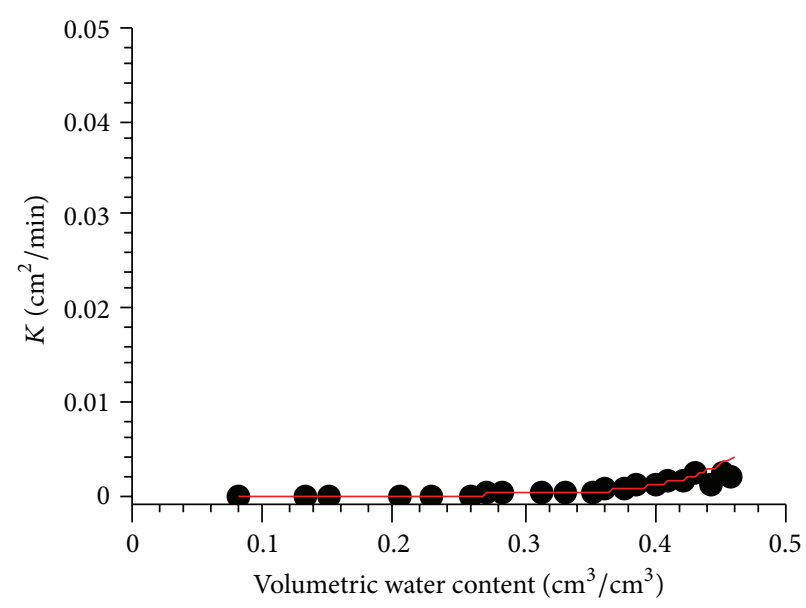

(d)

FIGURE 4: Unsaturated hydraulic conductivity of the four groups at 15 days. (a) Control group; (b) P-S1; (c) P-S2; (d) P-S3.

group's data at different times was 0.144 . By contrast, all the SAP-treated groups demonstrated significant decreases in unsaturated diffusivity during the early period of the experiment, and the rate of the decreases increased with SAP concentration, such that P-S1 > P-S2 > P-S3. The treatment groups' unsaturated diffusivities gradually increased with time and were nearly equal to the control group's at the end of the experiment. The population variances of the unsaturated diffusivity data of the treatment groups were between 0.387 and 0.398 .

Also, Figure 1 shows that soil water diffusion was significantly obstructed during the early period. At 0 days, the average decreases in unsaturated diffusivity were between $70.1 \%$ and $76.6 \%$. These values fell to between $30.6 \%$ and $46.9 \%$ at 5 days and reached $49.5 \%$ to $68.1 \%$ at 10 days. After repeated absorption and release of water, the SAP incurred some structural damage, and SAP water absorbency decreased with time. As a result, the effect on water diffusion in the soil gradually weakened. The average decrease in unsaturated diffusivity dropped to between $9.5 \%$ and $25.5 \%$ at 60 days and between $9.4 \%$ and $26.1 \%$ at 90 days. At 120 days, the unsaturated diffusivities of the treatment groups were very close to the control group, and the average decreases in unsaturated diffusivity were $1.2 \%$ to $16.0 \%$.

Table 1 presents the curves fitted to the unsaturated diffusivity data and their corresponding degrees of correlation (coefficient of determination, $R^{2}$ ) for the four groups at different times $(T=0,5,10,15,20,25,30,40,50,60,90$, and $120 \mathrm{~d}$ ). A comprehensive regression analysis of the data yielded time-varying functional equations for calculating the unsaturated diffusivity of soil under the effect of SAP. The regression equations show that the time-varying functions include the time variable $t$, and the goodness of fit of each regression attains extremely high significance $(P<0.01)$, indicating that these functions can accurately describe the relationship between time and the unsaturated diffusivity of soil under the effect of SAPs.

3.2. Effect of SAP on Soil Water Characteristic Curves. Figure 2 shows the soil water characteristic curves of the four sample groups at $0,15,30,50,90$, and 120 days. The trends of the curves suggest that the SAP improved the water retention properties of the treated soil during the early period. A comparison of the soil water content at different suction 
TABLE 1: Unsaturated diffusivity of four groups at different times and fitted time-varying functions.

\begin{tabular}{|c|c|c|c|c|}
\hline \multirow{2}{*}{ Time $(d)$} & \multicolumn{4}{|c|}{ Sample group } \\
\hline & CG & P-S1 & $\mathrm{P}-\mathrm{S} 2$ & $\mathrm{P}-\mathrm{S} 3$ \\
\hline \multirow{2}{*}{$T=0$} & $y=0.0394 e^{10.3 x}$ & $y=0.0813 e^{6.4727 x}$ & $y=0.0839 e^{5.9665 x}$ & $y=0.0871 e^{6.3951 x}$ \\
\hline & $R^{2}=0.89428$ & $R^{2}=0.906$ & $R^{2}=0.87945$ & $R^{2}=0.842$ \\
\hline \multirow{2}{*}{$T=5$} & $y=0.0476 e^{9.374 x}$ & $y=0.0226 e^{9.9152 x}$ & $y=0.0192 e^{10.363 x}$ & $y=0.0107 e^{11.545 x}$ \\
\hline & $R^{2}=0.88238$ & $R^{2}=0.90912$ & $R^{2}=0.9138$ & $R^{2}=0.92577$ \\
\hline \multirow{2}{*}{$T=10$} & $y=0.0298 e^{10.589 x}$ & $y=0.0841 e^{6.9179 x}$ & $y=0.0568 e^{6.7552 x}$ & $y=0.0521 e^{8.0808 x}$ \\
\hline & $R^{2}=0.85276$ & $R^{2}=0.82682$ & $R^{2}=0.87724$ & $R^{2}=0.80069$ \\
\hline \multirow{2}{*}{$T=15$} & $y=0.0389 e^{10.067 x}$ & $y=0.0312 e^{9.6729 x}$ & $y=0.0586 e^{7.5697 x}$ & $y=0.0582 e^{8.1997 x}$ \\
\hline & $R^{2}=0.89588$ & $R^{2}=0.88509$ & $R^{2}=0.85454$ & $R^{2}=0.86273$ \\
\hline \multirow{2}{*}{$T=20$} & $y=0.0341 e^{10.412 x}$ & $y=0.0525 e^{7.8393 x}$ & $y=0.0335 e^{8.8144 x}$ & $y=0.0561 e^{7.7954 x}$ \\
\hline & $R^{2}=0.88312$ & $R^{2}=0.84682$ & $R^{2}=0.90142$ & $R^{2}=0.79191$ \\
\hline \multirow{2}{*}{$T=25$} & $y=0.028 e^{10.676 x}$ & $y=0.0484 e^{8.2939 x}$ & $y=0.0235 e^{9.3367 x}$ & $y=0.0489 e^{8.5751 x}$ \\
\hline & $R^{2}=0.89384$ & $R^{2}=0.765$ & $R^{2}=0.90027$ & $R^{2}=0.82355$ \\
\hline \multirow{2}{*}{$T=30$} & $y=0.0332 e^{9.9538 x}$ & $y=0.0539 e^{8.3136 x}$ & $y=0.0194 e^{10.268 x}$ & $y=0.045 e^{8.6653 x}$ \\
\hline & $R^{2}=0.83787$ & $R^{2}=0.84295$ & $R^{2}=0.89589$ & $R^{2}=0.85636$ \\
\hline \multirow{2}{*}{$T=40$} & $y=0.0336 e^{10.385 x}$ & $y=0.045 e^{8.7363 x}$ & $y=0.0417 e^{8.2729 x}$ & $y=0.0336 e^{9.3069 x}$ \\
\hline & $R^{2}=0.85256$ & $R^{2}=0.84868$ & $R^{2}=0.83049$ & $R^{2}=0.87273$ \\
\hline \multirow{2}{*}{$T=50$} & $y=0.0232 e^{11.378 x}$ & $y=0.0217 e^{11.079 x}$ & $y=0.0129 e^{11.668 x}$ & $y=0.0204 e^{10.845 x}$ \\
\hline & $R^{2}=0.89875$ & $R^{2}=0.90882$ & $R^{2}=0.94285$ & $R^{2}=0.86495$ \\
\hline \multirow{2}{*}{$T=60$} & $y=0.0238 e^{11.387 x}$ & $y=0.0175 e^{11.924 x}$ & $y=0.023 e^{10.564 x}$ & $y=0.0285 e^{10.528 x}$ \\
\hline & $R^{2}=0.8949$ & $R^{2}=0.88716$ & $R^{2}=0.91468$ & $R^{2}=0.83412$ \\
\hline \multirow{2}{*}{$T=90$} & $y=0.0323 e^{10.511 x}$ & $y=0.028 e^{10.634 x}$ & $y=0.0253 e^{10.414 x}$ & $y=0.0135 e^{12.467 x}$ \\
\hline & $R^{2}=0.84247$ & $R^{2}=0.87298$ & $R^{2}=0.90481$ & $R^{2}=0.89243$ \\
\hline \multirow{2}{*}{$T=120$} & $y=0.0135 e^{12.467 x}$ & $y=0.0249 e^{10.86 x}$ & $y=0.025 e^{10.582 x}$ & $y=0.0259 e^{11.156 x}$ \\
\hline & $R^{2}=0.89243$ & $R^{2}=0.89367$ & $R^{2}=0.84869$ & $R^{2}=0.83914$ \\
\hline \multirow{2}{*}{ Overall } & $y=e^{10.3885 x-0.00093 t-3.3756}$ & $y=e^{8.80401 x+0.00289 t-3.42063}$ & $y=e^{8.7892 x+0.002612 t-3.20427}$ & $y=e^{8.9388 x+0.002882 t-3.27933}$ \\
\hline & $R^{2}=0.8709^{* *}$ & $R^{2}=0.857^{* *}$ & $R^{2}=0.8415^{* *}$ & $R^{2}=0.8185^{* *}$ \\
\hline
\end{tabular}

** indicated significant correlation at the 0.01 level.

TABLE 2: Unsaturated hydraulic conductivity at different times and fitted time-varying functions.

\begin{tabular}{|c|c|c|c|c|c|}
\hline \multirow{2}{*}{\multicolumn{2}{|c|}{ Time (d) }} & \multicolumn{4}{|c|}{ Sample group } \\
\hline & & CG & $\mathrm{P}-\mathrm{S} 1$ & P-S2 & P-S3 \\
\hline \multirow{2}{*}{\multicolumn{2}{|c|}{$T=0$}} & $y=1 E-08 e^{30.9 x}$ & $y=1 E-07 e^{20.96 x}$ & $y=2 E-07 e^{20.482 x}$ & $y=2 E-08 e^{26.611 x}$ \\
\hline & & $R^{2}=0.98014$ & $R^{2}=0.94428$ & $R^{2}=0.90433$ & $R^{2}=0.95688$ \\
\hline \multirow{2}{*}{\multicolumn{2}{|c|}{$T=15$}} & $y=7 E-08 e^{26.55 x}$ & $Y=4 E-08 e^{24.663 x}$ & $y=2 E-07 e^{20.871 x}$ & $y=1 E-07 e^{23.141 x}$ \\
\hline & & $R^{2}=0.9592$ & $R^{2}=0.96746$ & $R^{2}=0.94507$ & $R^{2}=0.93723$ \\
\hline \multirow{2}{*}{\multicolumn{2}{|c|}{$T=30$}} & $y=8 E-08 e^{24.229 x}$ & $y=1 E-07 e^{21.68 x}$ & $y=2 E-08 e^{25.708 x}$ & $y=4 E-08 e^{25.779 x}$ \\
\hline & & $R^{2}=0.96186$ & $R^{2}=0.95621$ & $R^{2}=0.93738$ & $R^{2}=0.96299$ \\
\hline \multirow{2}{*}{\multicolumn{2}{|c|}{$T=50$}} & $y=4 E-08 e^{28.525 x}$ & $y=3 E-08 e^{26.353 x}$ & $y=2 E-08 e^{27.173 x}$ & $y=3 E-08 e^{26.771 x}$ \\
\hline & & $R^{2}=0.97868$ & $R^{2}=0.94403$ & $R^{2}=0.97353$ & $R^{2}=0.96353$ \\
\hline \multirow{2}{*}{\multicolumn{2}{|c|}{$T=90$}} & $y=6 E-08 e^{25.988 x}$ & $y=4 E-08 e^{25.597 x}$ & $y=3 E-08 e^{26.226 x}$ & $y=1 E-08 e^{28.494 x}$ \\
\hline & & $R^{2}=0.96934$ & $R^{2}=0.95079$ & $R^{2}=0.95412$ & $R^{2}=0.9598$ \\
\hline \multirow{2}{*}{\multicolumn{2}{|c|}{$T=120$}} & $y=2 E-08 e^{27.958 x}$ & $y=4 E-08 e^{25.532 x}$ & $Y=3 E-08 e^{26.751 x}$ & $y=2 E-08 e^{29.285 x}$ \\
\hline & & $R^{2}=0.96096$ & $R^{2}=0.97013$ & $R^{2}=0.94311$ & $R^{2}=0.91343$ \\
\hline & Parameter & \multicolumn{4}{|c|}{$y=e^{a x+b t+c x^{2}+d t^{2}+f}$} \\
\hline \multirow{6}{*}{ Overall } & $a$ & $4.02 E+01$ & $5.49 E+01$ & $4.67 E+01$ & $5.17 E+01$ \\
\hline & $b$ & $1.80 E-03$ & $2.74 E-03$ & $8.14 E-03$ & $-2.16 E-03$ \\
\hline & $c$ & $-2.08 E+01$ & $-4.98 E+01$ & $-3.71 E+01$ & $-4.29 E+01$ \\
\hline & $d$ & $-5.62 E-05$ & $-8.69 E-06$ & $-3.88 E-05$ & $1.62 E-05$ \\
\hline & $f$ & $-1.86 E+01$ & $-2.11 E+01$ & $-2.00 E+01$ & $-2.04 E+01$ \\
\hline & $R^{2}$ & $0.9659^{* *}$ & $0.9708^{* *}$ & $0.9702^{* *}$ & $0.9702^{* *}$ \\
\hline
\end{tabular}

** indicated significant correlation at the 0.01 level. 


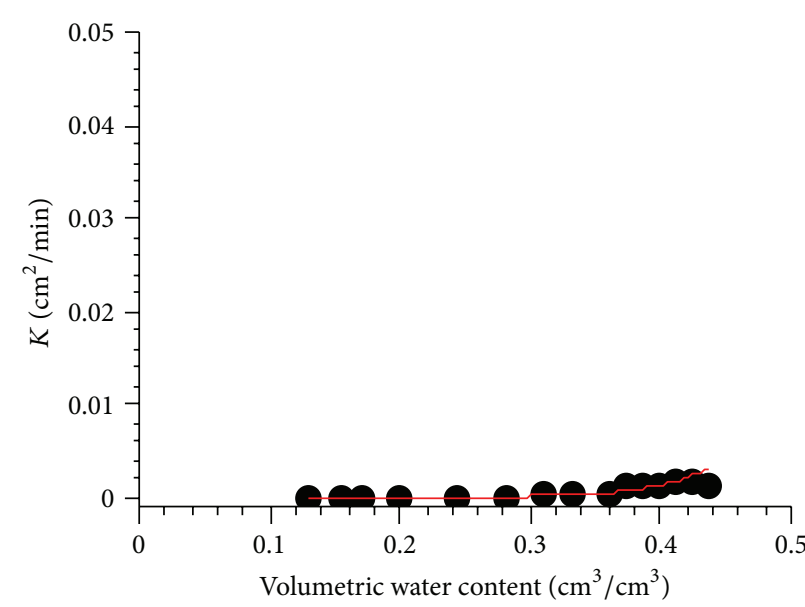

(a)

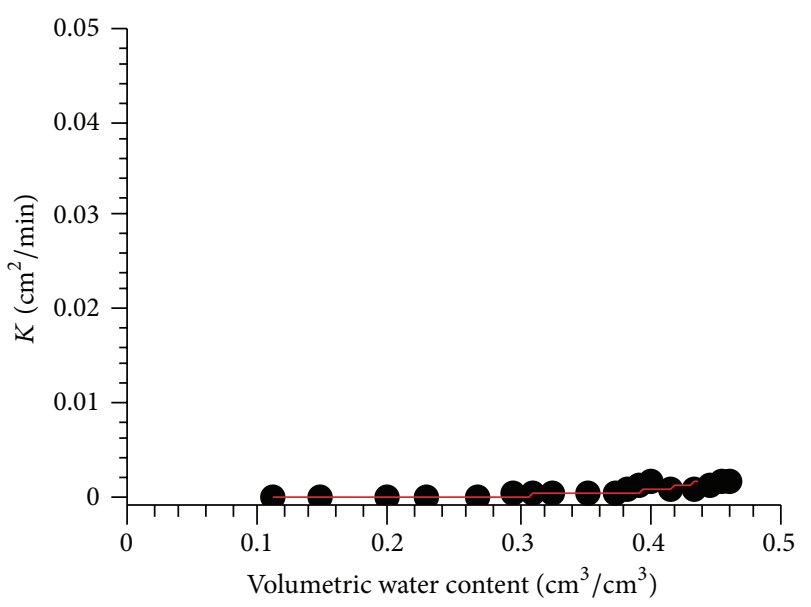

(c)

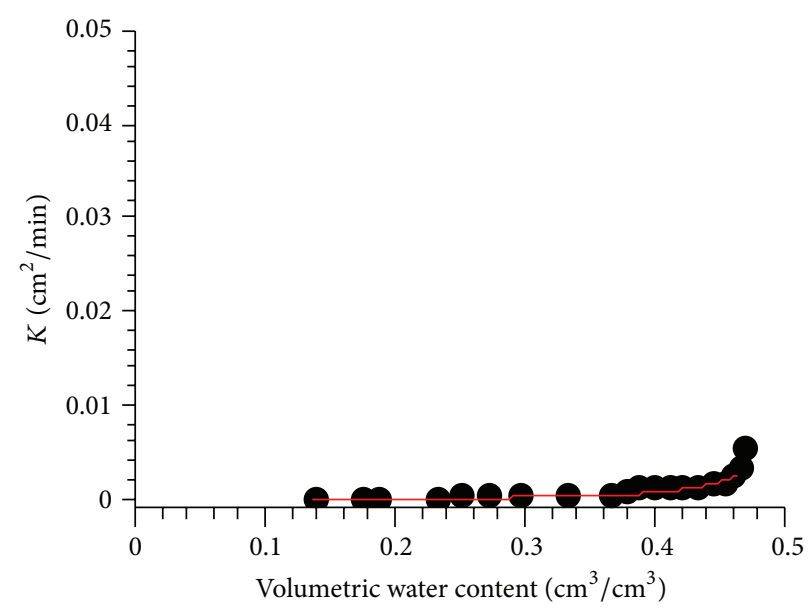

(b)

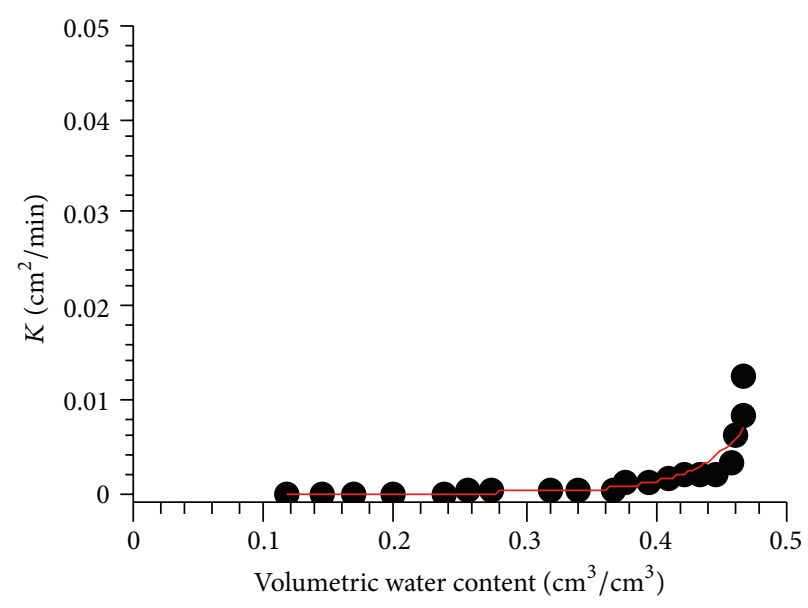

(d)

FIGURE 5: Unsaturated hydraulic conductivity of the four groups at 30 days. (a) Control group; (b) P-S1; (c) P-S2; (d) P-S3.

pressure ranges demonstrated that the improvement in soil water retention mainly occurred in the suction pressure range of 0 to $3,000 \mathrm{~cm}$, and it was especially marked in the 100 to $800 \mathrm{~cm}$ range. At 0 days, the soil in the P-S1, P-S2, and P-S3 groups showed water content increases of $10.6 \%$ to $26.4 \%, 14.2 \%$ to $17.0 \%$, and $7.7 \%$ to $10.6 \%$, respectively, compared to the control group; the P-S1 group experienced the increase of $26.4 \%$ at a suction pressure of $300 \mathrm{~cm}$. By 15 days, the soil water content in the P-S1, P-S2, and P-S3 groups increased by $15.4 \%$ to $26.5 \%, 14.8 \%$ to $21.6 \%$, and $9.7 \%$ to $14.7 \%$, respectively; the largest increase of $26.5 \%$ occurred in the P-S1 group at a suction pressure of $500 \mathrm{~cm}$. After a duration of 30 days, the soil water content in the three treatment groups increased by $11.8 \%$ to $20.7 \%, 10.2 \%$ to $14.4 \%$, and $5.1 \%$ to $9.2 \%$, respectively, with the largest increase of $20.7 \%$ occurring in the P-S1 group at a suction pressure of $500 \mathrm{~cm}$. By 50 days, the soil water content increases in each of the three treatment groups were $8.3 \%$ to $23.2 \%, 12.4 \%$ to $18.3 \%$, and $8.8 \%$ to $14.1 \%$, respectively; the maximum of $23.2 \%$ occurred in the P-S1 group at a suction pressure of $100 \mathrm{~cm}$. By 90 days, these values were $10.3 \%$ to $13.0 \%, 7.3 \%$ to $11.6 \%$, and $7.1 \%$ to $10.4 \%$, with the maximum of $13.0 \%$ occurring in the
P-S1 group at a suction pressure of $500 \mathrm{~cm}$. At 120 days, the soil water content in the treatment groups was only $5.6 \%$ to $7.7 \%, 3.4 \%$ to $4.0 \%$, and $2.7 \%$ to $4.2 \%$ higher than in the control group, and the P-S1 group saw the highest soil water content increase of $7.7 \%$ at a suction pressure of $100 \mathrm{~cm}$. Those soil water characteristic curves were used for predicting specific moisture capacity $C(\theta)$ to calculate unsaturated hydraulic conductivities of the soil. Finally, timevarying functions for the unsaturated hydraulic conductivity under the effect of SAP were derived from a series of calculated unsaturated hydraulic conductivities.

\subsection{Effect of SAP on Unsaturated Hydraulic Conductivity.} Figures 3-8 show the unsaturated hydraulic conductivities of the control group and the treatment groups at $0,15,30,50$, 90 , and 120 days, respectively. Compared to the control group, we can see the unsaturated hydraulic conductivities of treated soil sample were greatly decreased after SAP application during the early period, similar to the effect of SAP on unsaturated diffusivity. The average decreases in unsaturated hydraulic conductivity were $85.5 \%$ to $94.1 \%$ at 0 days, $75.1 \%$ to $82.9 \%$ at 30 days, and $65.6 \%$ to $76.2 \%$ at 50 days. Notably, 


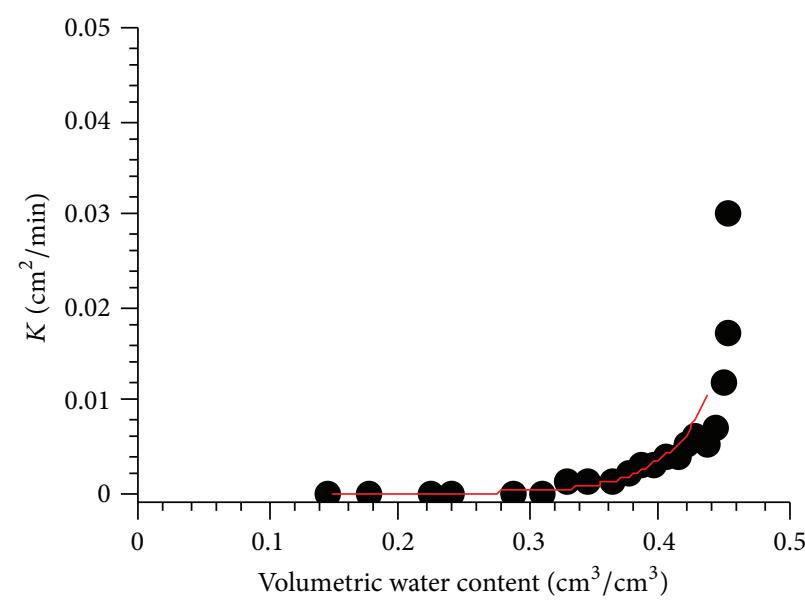

(a)

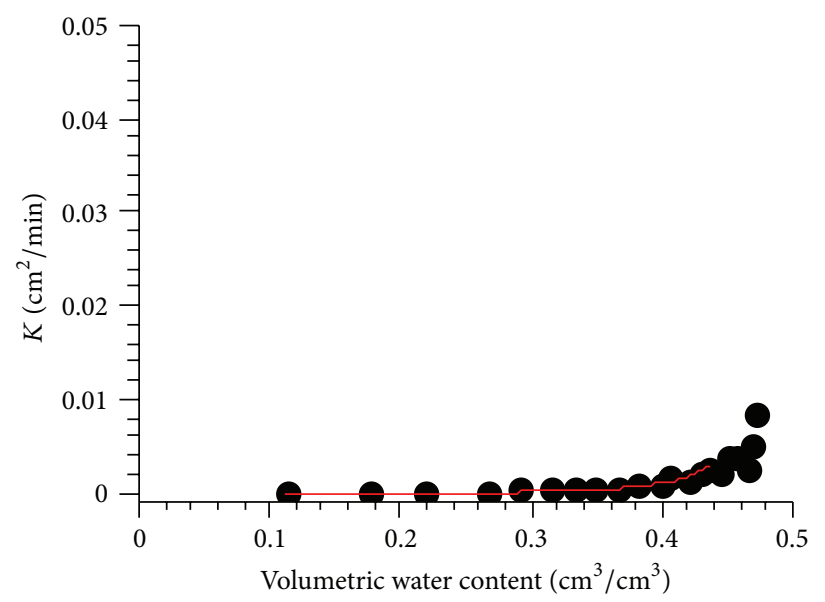

(c)

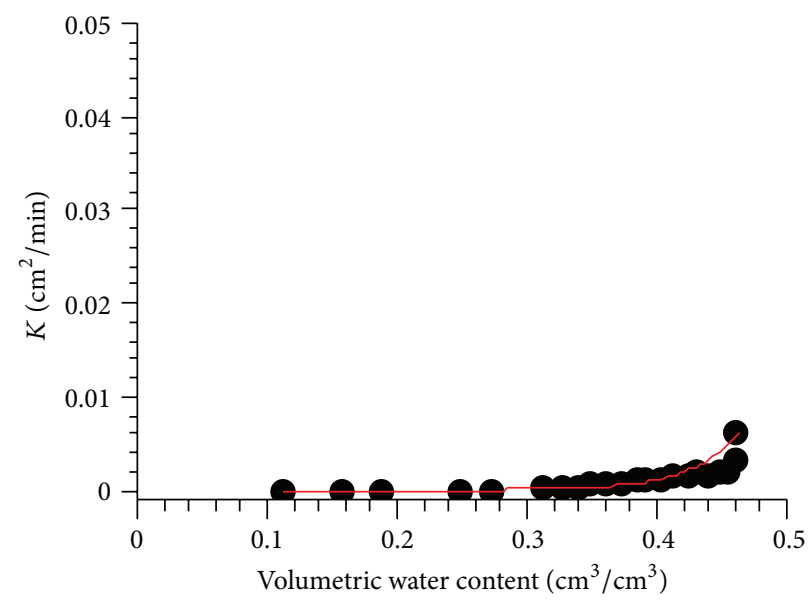

(b)

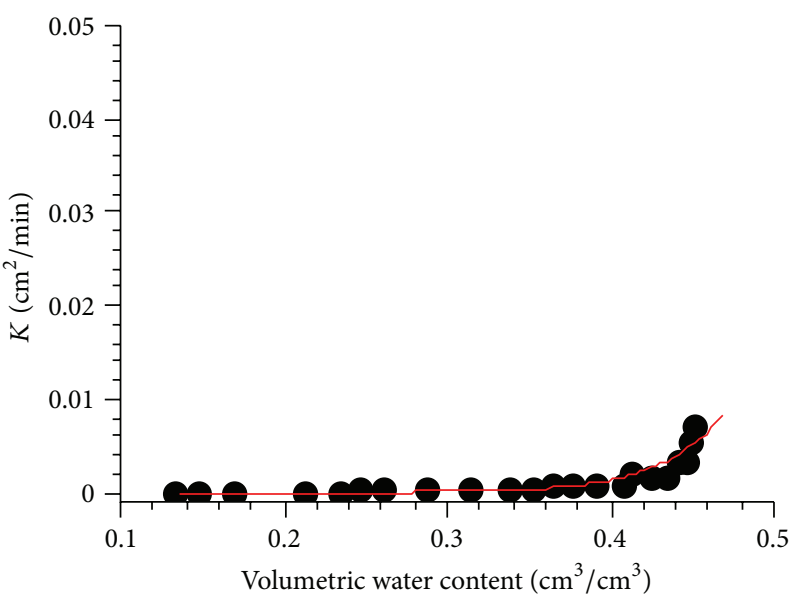

(d)

FIgURE 6: Unsaturated hydraulic conductivity of the four groups at 50 days. (a) Control group; (b) P-S1; (c) P-S2; (d) P-S3.

abnormalities were observed in regions with high water content in all treatment groups at 30,90, and 120 days. It was therefore impossible to calculate $K$ values for these regions.

Table 2 displays the curves fitted to unsaturated hydraulic conductivity data at different times $(T=0,15,30,50,90$, and $120 \mathrm{~d}$ ), as well as corresponding degrees of correlation. Timevarying functional equations describing soil's unsaturated hydraulic conductivity were constructed through a comprehensive regression analysis. As the regression equations show, these functions include the time variable $t$, and each regression's goodness of fit attained extremely high significance $(P<0.01)$, demonstrating that these functions can precisely reflect the relationships of unsaturated hydraulic conductivity varying with the test time under the influence of SAP.

\section{Discussion}

4.1. Water Retention Properties of SAP-Treated Soil. Normally, the water retention properties of soil are closely correlated with the pore size, while the water characteristic curve of a soil sample represents the relationship between its porosity and water content. When soil is treated with SAP, its water retention properties are related to both porosity and the SAP's water absorbency. An in-depth analysis of the SAP-treated soil in this study revealed that the SAP had the greatest effect on water retention in soil sections with suction pressure between 100 and $800 \mathrm{~cm}$, because these areas exhibited the greatest increases in water content compared to the control group and other sections in the treatment groups. This result was generally consistent with the conclusion drawn by Han [23], who stated that SAPs have the most dramatic water conservation effects in soil sections with suction pressure between 0 and $1000 \mathrm{~cm}$. It is widely accepted that water stored in the suction pressure range of 0 to $15,000 \mathrm{~cm}$ is available water that can be used by plants, and $50 \%$ to $70 \%$ of the available water content is retained in the suction pressure range of 0 to $800 \mathrm{~cm} \mathrm{[24].} \mathrm{Therefore,} \mathrm{the} \mathrm{water} \mathrm{held} \mathrm{by} \mathrm{SAPs}$ in this range was considered to be available for growing plants in this study.

4.2. Hydraulic Parameters of SAP-Treated Soils. The Richards equation-based modeling of soil water movement relies on functions that can accurately characterize a soil's hydraulic parameters. Normally, the water diffusivity of unsaturated soil only depends on its water content $(\theta)$; however, SAPtreated soil's unsaturated diffusivity is strongly correlated 


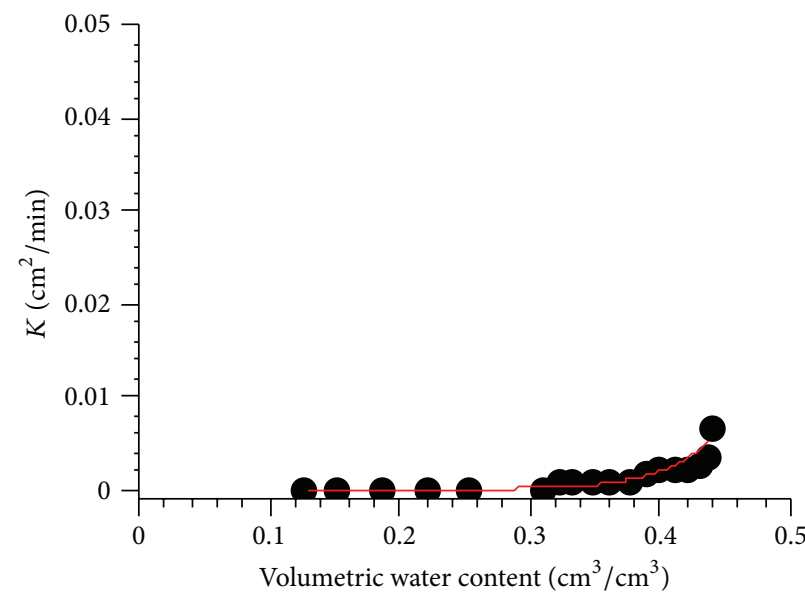

(a)

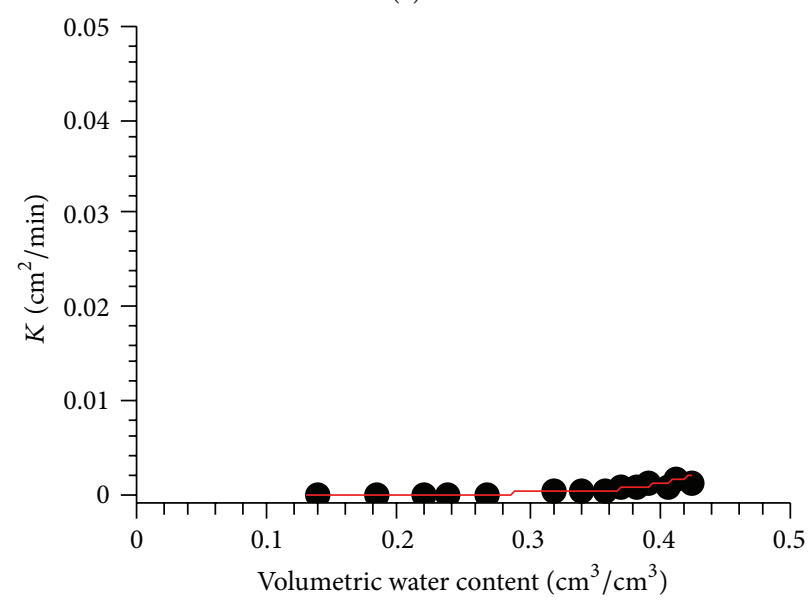

(c)

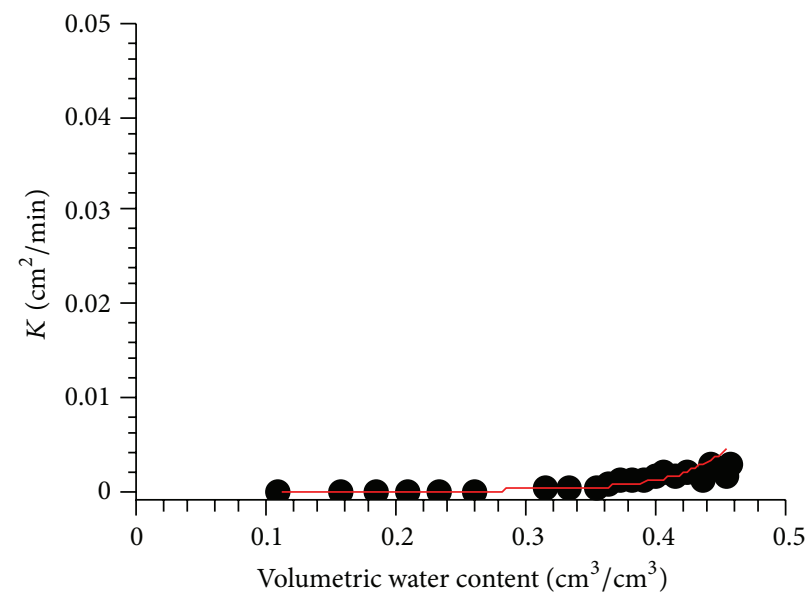

(b)

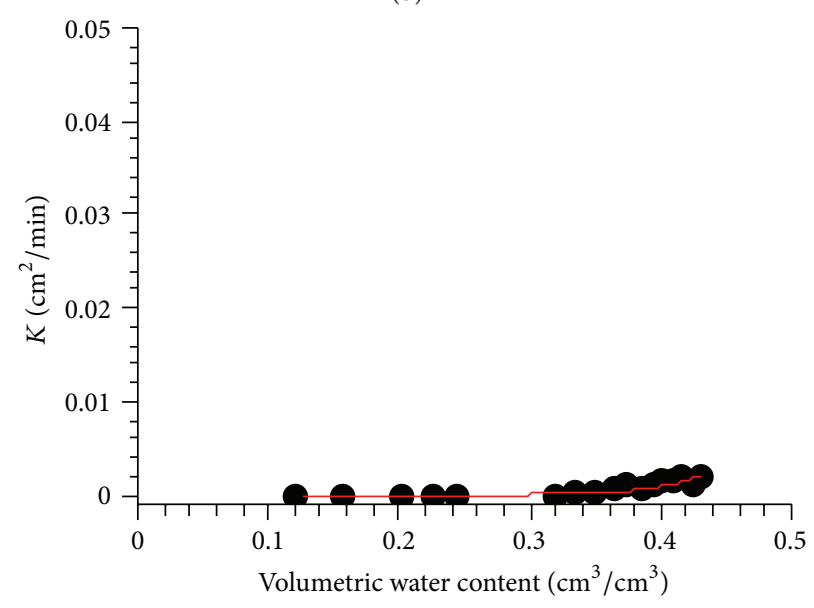

(d)

FIGURE 7: Unsaturated hydraulic conductivity of the four groups at 90 days. (a) Control group; (b) P-S1; (c) P-S2; (d) P-S3.

with the duration of the SAP's presence in the soil $(t)$, in addition to soil water content $(\theta)$. The experimental results presented in this paper suggest that the SAP had a strong effect on water diffusion during the early testing period. However, because the SAP's water-absorbing membrane structure was gradually damaged by microbial decomposition and electrostatic interactions with highly charged ions, fertilizers, and pesticides dissolved in the soil solution $[25,26]$, the effects of enhancing soil water retention and obstructing soil water movement weakened with time, consistent with the research finding by Han et al. [12]. This suggests that the hydraulic parameters of SAP-treated soil are time-varying and dynamic, a finding that should be fully considered in constructing water movement models.

The variation in unsaturated hydraulic conductivity $K$ under the effect of SAP was similar to the variation in unsaturated diffusivity $D$, and the fitted functions were also functions of both water content $\theta$ and duration $t$. In this paper, $K$ values were estimates obtained from the measurements of $D$ according to the formula $\theta=\theta_{r}+\left(\theta_{s}-\theta_{r}\right) /\left[1+(\alpha h)^{n}\right]^{m}$ rather than real data. The experimental results indicate that when the difference between the fitted saturated water content $\left(\theta_{s}\right)$ and fitted wilting point $\left(\theta_{r}\right)$ was smaller than the difference between the measured water content $(\theta)$ and fitted wilting point $\left(\theta_{r}\right)$, error could occur in calculating the hydraulic head $(h)$, thus impeding the accurate characterization of $K$ values in regions with high water content. The lack of $K$ values in these regions has an adverse impact on the accuracy of the fitted time-varying functions; therefore, special attention should be paid to this phenomenon in modeling. The aforementioned error can be reduced by improving the stability and precision of test instruments, such as high-speed centrifuge and electronic scale.

\section{Conclusions}

(1) During the early testing period, water diffusion in the SAP-treated soil samples was significantly impacted by the presence of SAP. During the period from 0 to 10 days, the average decreases in unsaturated diffusivity decreased were measured at between $30.6 \%$ and $76.6 \%$. As the SAP's water absorbency declined over time, the effect of obstructing soil water movement gradually weakened. During the period between 60 and 120 days, the average decreases in unsaturated diffusivity fell to $1.2 \%$ to $26.1 \%$. In contrast, the 


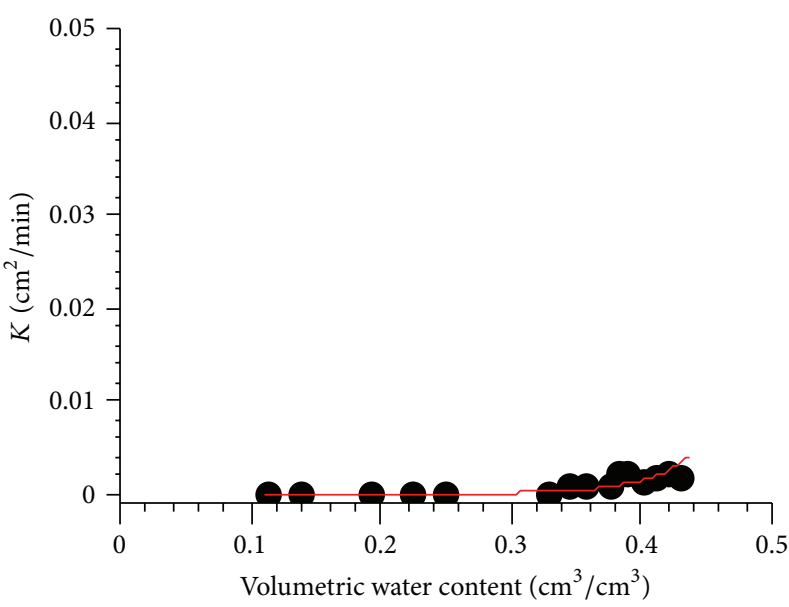

(a)

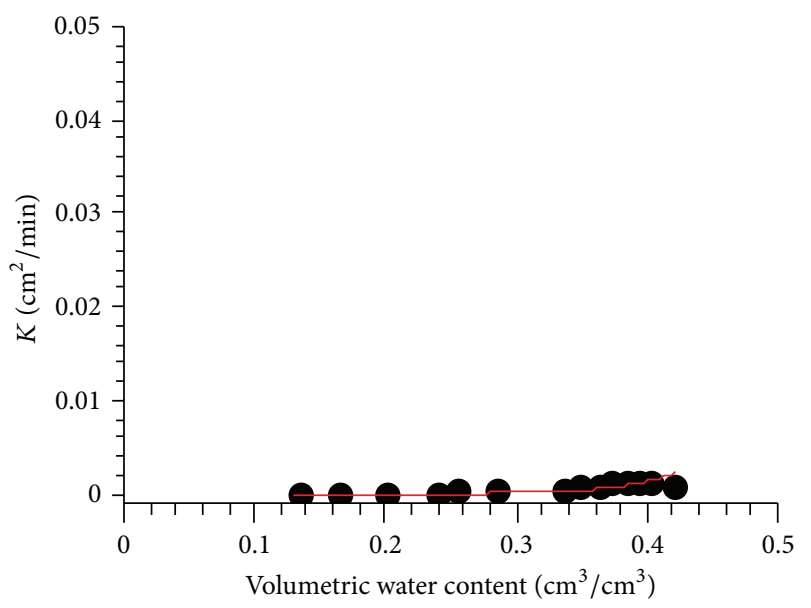

(c)

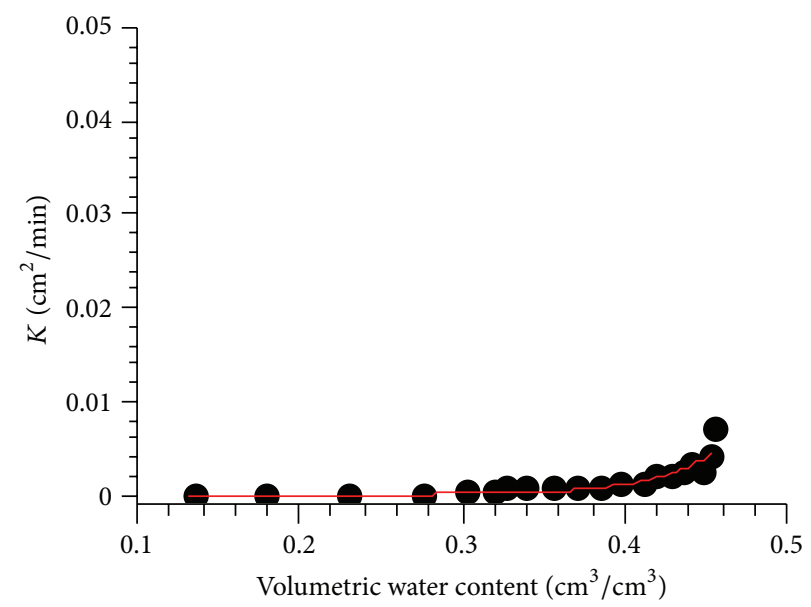

(b)

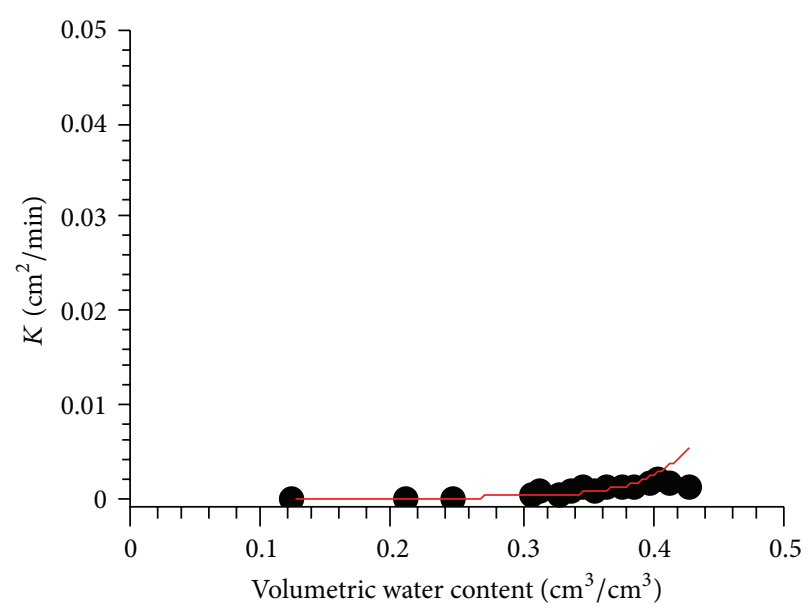

(d)

FIGURE 8: Unsaturated hydraulic conductivity of the four groups at 120 days. (a) Control group; (b) P-S1; (c) P-S2; (d) P-S3.

unsaturated diffusivity of the control group exhibited little variation throughout the experiment; the population variance of the control group's data at different times was 0.144 , compared to 0.387 to 0.398 for the treatment groups.

(2) The experimental SAP greatly decreased the treated soil samples' unsaturated hydraulic conductivities during the early period, similar to its effect on unsaturated diffusivity. During the period between 0 and 50 days, the average decreases in unsaturated hydraulic conductivity were $65.6 \%$ to $94.1 \%$. The $K$ values obtained based on the measurement of $D$ and the formula $\theta=\theta_{r}+\left(\theta_{s}-\theta_{r}\right) /\left[1+(\alpha h)^{n}\right]^{m}$ were dramatically affected by the accuracy of the fitted parameters of the soil water characteristic curve.

(3) The experimental SAP improved the water retaining capacity of soil sections where the suction pressure was between 0 and $3,000 \mathrm{~cm}$, resulting in an average increase of $2.7 \%$ to $26.5 \%$ in soil water content in the treatment groups. The improvement in soil water retention was especially marked in sections with suction pressure ranging from 100 to $800 \mathrm{~cm}$, and the water stored in this section was considered to be available water.

\section{Competing Interests}

The authors declare that there is no conflict of interests regarding the publication of this paper.

\section{Acknowledgments}

This work was financially supported by the National Natural Science Foundation of China (51379210), National Science and Technology Project (2015BAD20B03), and Beijing Science and Technology Plan (D151100004115002).

\section{References}

[1] A. Egrinya Eneji, R. Islam, P. An, and U. C. Amalu, "Nitrate retention and physiological adjustment of maize to soil amendment with superabsorbent polymers," Journal of Cleaner Production, vol. 52, no. 4, pp. 474-480, 2013. 
[2] Y. G. Han, P. L. Yang, S. M. Ren, H. Liu, and Y. Bi, "Effects of super absorbent polymers on water saving and irrigation schedule for apple irrigation," Transactions of the Chinese Society of Agricultural Engineering, vol. 22, no. 9, pp. 70-73, 2006.

[3] H. Namvar, A. Asgharzade, M. Babaeian et al., "The effects of superabsorbent polymer on yield and yield component of two grape varieties," International Journal of Biosciences, vol. 4, no. 4, pp. 18-23, 2014.

[4] W. Zhuang, P. Wu, H. Feng, F. Xu, B. Li, and R. Ning, "Effects of super absorbent polyer of sodium polyacrylate used in soil on the growth and yield of winter wheat," Transactions of the Chinese Society of Agricultural Engineering, vol. 24, no. 5, pp. 3741, 2008.

[5] N. C. Turner, "Agronomic options for improving rainfall-use efficiency of crops in dryland farming systems," Journal of Experimental Botany, vol. 55, no. 407, pp. 2413-2425, 2004.

[6] X. Wang, D. Cai, H. Wu, W. B. Hoogmoed, and O. Oenema, "Effects of variation in rainfall on rainfed crop yields and water use in dryland farming areas in China," Arid Land Research and Management, vol. 30, no. 1, pp. 1-24, 2016.

[7] M. C. McCutcheon, H. J. Farahani, J. D. Stednick, G. W. Buchleiter, and T. R. Green, "Effect of soil water on apparent soil electrical conductivity and texture relationships in a dryland field," Biosystems Engineering, vol. 94, no. 1, pp. 19-32, 2006.

[8] P. L. Yang, R. K. Liao, S. M. Ren, and Y. Li, "Application of chemical regulating technology for utilization of water and fertilizer in dry-land agriculture," Transactions of the Chinese Society for Agricultural Machinery, vol. 44, no. 6, pp. 100-109, 2013.

[9] L. Yang, Y. Yang, Z. Chen, C. Guo, and S. Li, "Influence of super absorbent polymer on soil water retention, seed germination and plant survivals for rocky slopes eco-engineering," Ecological Engineering, vol. 62, pp. 27-32, 2014.

[10] R. K. Liao, P. L. Yang, and S. M. Ren, "Review on super absorbent polymer application for improving fertilizer efficiency and controlling agricultural non-point source pollutions," Transactions of the Chinese Society of Agricultural Engineering, vol. 28, no. 17, pp. 1-10, 2012.

[11] F. Liu, H. Ma, S. Xing, Z. Du, B. Ma, and D. Jing, "Effects of super-absorbent polymer on dry matter accumulation and nutrient uptake of Pinus pinaster container seedlings," Journal of Forest Research, vol. 18, no. 3, pp. 220-227, 2013.

[12] Y. Han, X. Yu, P. Yang, B. Li, L. Xu, and C. Wang, "Dynamic study on water diffusivity of soil with super-absorbent polymer application," Environmental Earth Sciences, vol. 69, no. 1, pp. 289-296, 2013.

[13] W. Bai, H. Zhang, B. Liu, Y. Wu, and J. Q. Song, "Effects of superabsorbent polymers on the physical and chemical properties of soil following different wetting and drying cycles," Soil Use \& Management, vol. 26, no. 3, pp. 253-260, 2010.

[14] X. Li, J.-Z. He, J. M. Hughes, Y.-R. Liu, and Y.-M. Zheng, "Effects of super-absorbent polymers on a soil-wheat (Triticum aestivum L.) system in the field," Applied Soil Ecology, vol. 73, pp. 58-63, 2014.

[15] Y. G. Han, P. L. Yang, Y. P. Luo, S. M. Ren, L. X. Zhang, and L. Xu, "Porosity change model for watered super absorbent polymertreated soil," Environmental Earth Sciences, vol. 61, no. 6, pp. 1197-1205, 2010.

[16] M. R. Guilherme, F. A. Aouada, A. R. Fajardo et al., "Superabsorbent hydrogels based on polysaccharides for application in agriculture as soil conditioner and nutrient carrier: a review," European Polymer Journal, vol. 72, pp. 365-385, 2015.
[17] W. H. Zhuang, H. Feng, and P. T. Wu, "Development of super absorbent polymer and its application in agriculture," Transactions of the Chinese Society of Agricultural Engineering, vol. 23, no. 6, pp. 265-270, 2007.

[18] D. Russo, "Determining soil hydraulic properties by parameter estimation: on the selection of a model for the hydraulic properties," Water Resources Research, vol. 24, no. 3, pp. 453459, 1988.

[19] J. Zhu and B. P. Mohanty, "Soil hydraulic parameter upscaling for steady-state flow with root water uptake," Vadose Zone Journal, vol. 3, no. 4, pp. 1464-1470, 2004.

[20] A. V. M. Ines and B. P. Mohanty, "Parameter conditioning with a noisy Monte Carlo genetic algorithm for estimating effective soil hydraulic properties from space," Water Resources Research, vol. 44, no. 8, Article ID W08441, pp. 853-861, 2008.

[21] Y. G. Han, W. U. Heng-Fei, P. L. Yang et al., "Dynamic effects of super absorbent polymer on physical properties and water infiltration of soil," Agricultural Research in the Arid Areas, vol. 31, no. 5, pp. 161-167, 2013.

[22] S. Banedjschafie and W. Durner, "Water retention properties of a sandy soil with superabsorbent polymers as affected by aging and water quality," Journal of Plant Nutrition and Soil Science, vol. 178, no. 5, pp. 798-806, 2015.

[23] Y. G. Han, The Principle of Typical Chemical Water-Saving Preparation and the Research of Their Integrated Application, China Agricultural University, Beijing, China, 2007.

[24] M. A. Shao, Q. J. Wang, and M. B. Huang, Soil Physics, Higher Education Press, Beijing, China, 2006.

[25] B. Zhou, R. K. Liao, Y. K. Li et al., "Water-absorption characteristics of organic-inorganic composite superabsorbent polymers and its effect on summer maize root growth," Journal of Applied Polymer Science, vol. 126, no. 2, pp. 423-435, 2012.

[26] R. K. Liao, S. M. Ren, and P. L. Yang, "Quantitative fractal evaluation of herbicide effects on the water-absorbing capacity of superabsorbent polymers," Journal of Nanomaterials, vol. 2014, Article ID 905630, 9 pages, 2014. 

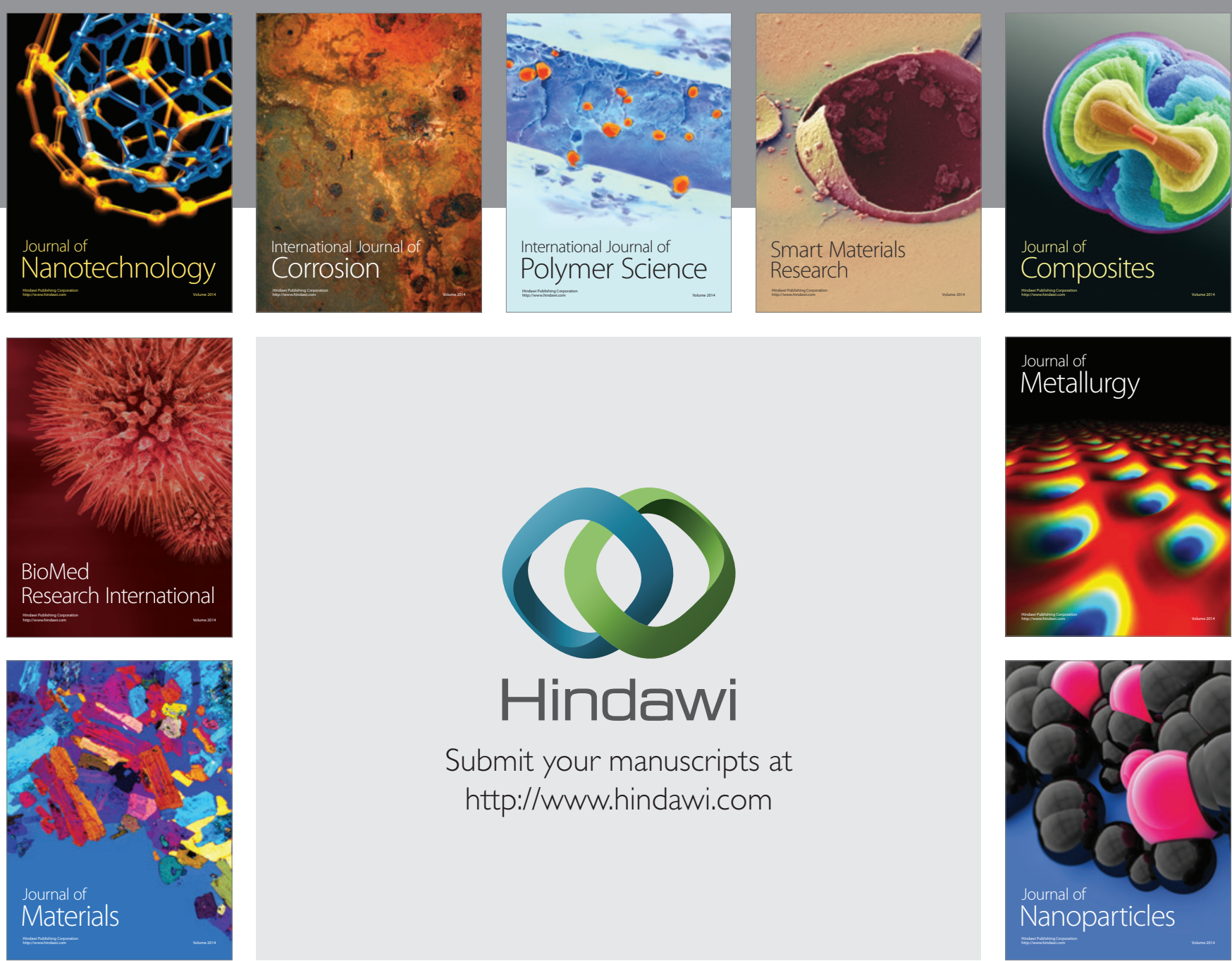

\section{Hindawi}

Submit your manuscripts at

http://www.hindawi.com

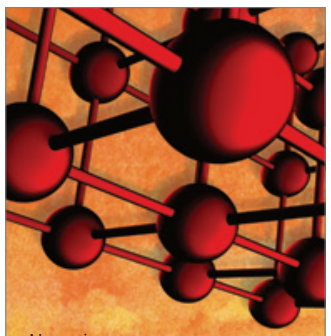

Materials Science and Engineering
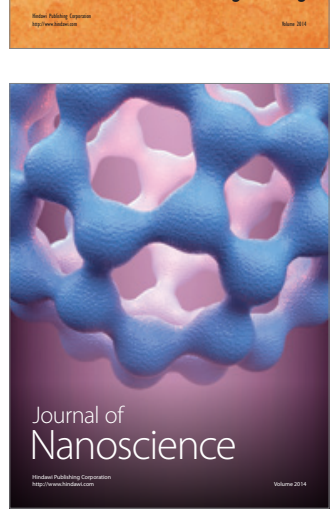
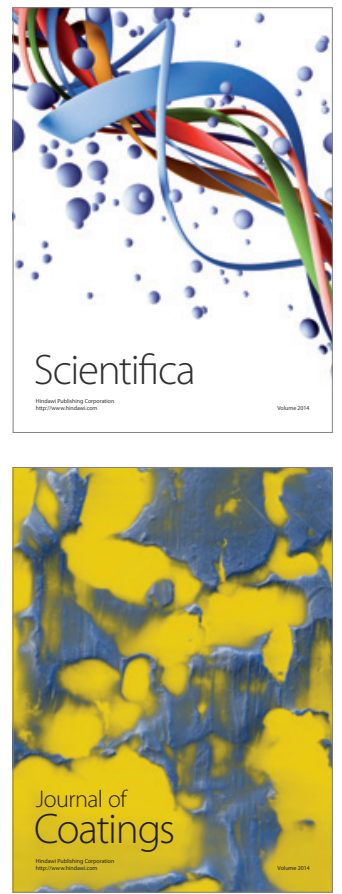
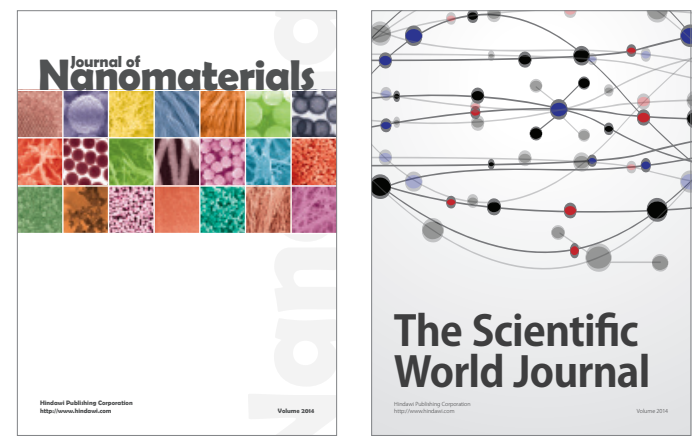

The Scientific World Journal
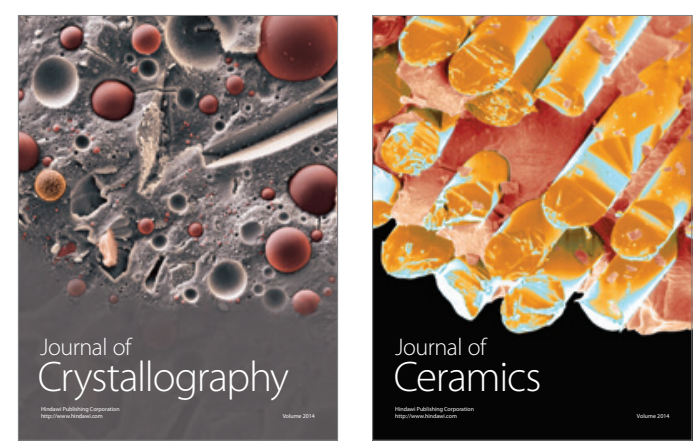
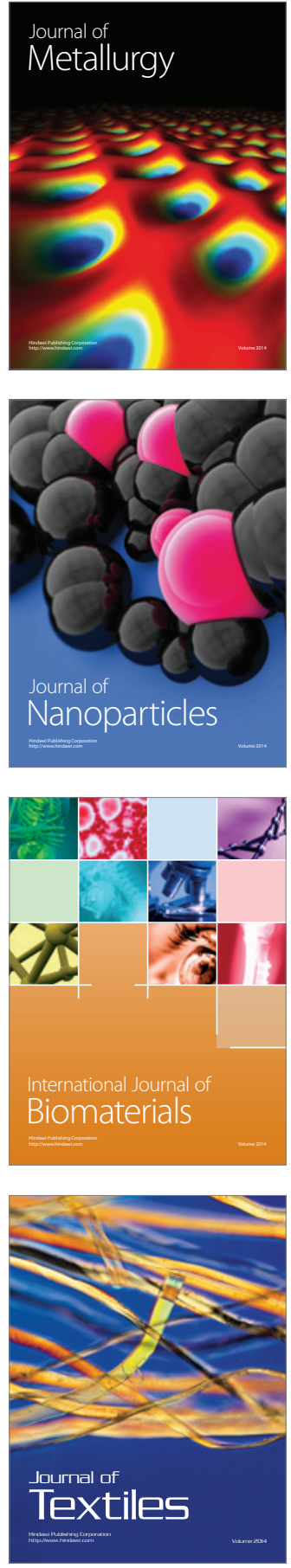